\title{
Tibial and Common Fibular Nerve Block in the Popliteal Fossa with Single Puncture Using Percutaneous Nerve Stimulator: Anatomical Considerations and Ultrasound Description
}

\author{
Viviane de Oliveira Rangel 1, Raphael de Almeida Carvalho 2, Beatriz Lemos da Silva Mandim, TSA ${ }^{3}$, \\ Rodrigo Rodrigues Alves ${ }^{2}$, Roberto Araújo Ruzi, TSA ${ }^{4}$, Neuber Martins da Fonseca, TSA 5
}

\begin{abstract}
Summary: Rangel VO, Carvalho RA, Mandim BLS, Alves RR, Ruzi RA, Fonseca NM - Tibial and Common Fibular Nerve Block in the Popliteal Fossa with Single Puncture Using Percutaneous Nerve Stimulator: Anatomical Considerations and Ultrasound Description.

Background and objectives: Techniques of peripheral nerve block have gained popularity over the last two decades becoming a growing anesthetic option for limb surgeries. This study proposes a technical approach of the tibial and common fibular nerves in the popliteal fossa with single puncture using percutaneous nerve stimulator, considering the correlation with an anatomical and ultrasound study.

Methods: This prospective, observational, randomized study was performed with 28 patients scheduled for foot surgeries. After localizing the tibial and common fibular nerves through percutaneous stimulation, the puncture was performed at the point of tibial nerve stimulation with a 5-cm needle (B.Braun, Stimuplex 50), and $10 \mathrm{~mL}$ of levobupivacaine were injected. The needle was pulled back and redirected to the point of common fibular nerve stimulation looking for the corresponding motor response, and $10 \mathrm{~mL}$ of the local anesthetic were injected. Imaging study of the popliteal region was performed by ultrasound to correlate the anatomy with the technique used.
\end{abstract}

Results: Adequate anesthesia was obtained in all cases. The mean time to localize the tibial and common fibular nerves suing the percutaneous stimulator was 57.1 and 32.8 seconds, respectively, and with the nerve stimulator it was 2.22 and 1.79 minutes, respectively. The mean depth of the needle into the tibial nerve was $10.7 \mathrm{~mm}$.

Conclusions: The approach for tibial and common fibular nerves with single puncture in the popliteal fossa using peripheral nerve stimulator is a good option for anesthesia and analgesia for foot surgeries.

Keywords: Transcutaneous Electric Nerve Stimulation; Tibial Nerve; Peroneal Nerve; Ultrasonography, Interventional; Anesthesia, conduction.

\section{INTRODUCTION}

Peripheral nerve block techniques have gained popularity over the last two decades, becoming increasingly the anesthetic choice for limb surgeries ${ }^{1}$. The introduction of peripheral nerve stimulators and more recently percutaneous nerve stimulator, in addition to ultrasound-guided approaches along with devices that allow continuous block with long-acting local

Received from Universidade Federal de Uberlândia (UFU), Brazil.

1. R3 at SBA/CET of the UFU

2. Anesthesiologist of Hospital de Clínicas da Faculdade de Medicina da UFU

3. Co-responsible for CET/SBA of Faculdade de Medicina da UFU

4. Co-responsible for CET/SBA of Faculdade de Medicina da UFU; Member of the Comitê

de Anestesia Locorregional

5. Professor of Anesthesiology; Responsible for CET/SBA of Faculdade de Medicina da

UFU; President of the Comissão de Normas Técnicas/SBA

Submitted on January 15, 2011.

Approved on February 21, 2011

Correspondence to:

Dra. Viviane de Oliveira Rangel

Rua Santa Catarina, 849, ap. 202

38400-652 - Uberlândia, MG, Brazil

E-mail: olirangelbr@yahoo.com.br anesthetics and better safety profile have contributed for this popularity making the use of those blocks attractive for outpatient procedures ${ }^{2,3}$.

The approaches in the popliteal fossa originally described for the posterior ${ }^{4}$ and lateral ${ }^{5}$ approaches have been consolidated in clinical practice. However, failures of one of the components of the sciatic nerve (tibial or common fibular) ${ }^{6-8}$, due to anatomical variations in the division of the sciatic nerve $6,7,9$, have been described motivating discussions on the need of simple or double stimulation on the posterior or lateral approach to reduce the number of failures 8,10 .

Percutaneous electrical stimulation allows localizing superficial nerves and it has been used in brachial plexus and femoral nerve blocks ${ }^{11,12}$. Better nerve identification reduces patient discomfort and improves the blockade efficacy due to the lower number of failures ${ }^{11}$. Recently, ultrasound-guidance to the popliteal fossa has been described for the classical posterior and lateral approaches ${ }^{13-17}$ with a high success rate.

The objective of this study was to propose a new approach to tibial and common fibular nerves in the popliteal fossa with single puncture using percutaneous nerve stimulator and peripheral nerve stimulator according to a prior ultrasound description to evaluate the anatomy of the region. 


\section{METHODS}

After approval by the Research Ethics Committee of Hospital das Clínicas da Faculdade de Medicina da Universidade Federal de Uberlândia and signing of the informed consent, 28 individuals of both genders, physical status ASA I to III, 19 to 78 years of age, weight 49 to $95 \mathrm{~kg}$, scheduled for foot surgery with or without pneumatic garroting were enrolled in this prospective observational study.

Patients with contraindications to regional anesthesia, with psychiatric diseases, or drug abuse were excluded.

Before the anesthetic blockade, a venous access was performed with a 20G catheter into a vein in the forearm for the infusion of crystalloids. Monitoring consisted of continuous electrocardioscope, pulse oximetry, and automatic non-invasive blood pressure during the blockade and perioperatively. The blockade was performed after sedation with 1 to $3 \mathrm{mg}$ of intravenous midazolam (IV).

All patients were placed in horizontal dorsal decubitus position with hip joint at $90^{\circ}$ flexion and knee joint at $120^{\circ}$ flexion with the help of an assistant (Figure 1).

Anatomical references used in the procedure were obtained through an ultrasound study of the popliteal region in three patients to correlate the anatomy with the proposed technique. A line between the tendons of the femoral biceps and tendons of the semitendinosus and semimembranosus muscle was drawn on the popliteal fold. Percutaneous pre-localization of the tibial nerve was performed in the middle of this line with an initial current intensity of $4 \mathrm{~mA}$ and stimulus duration of $1 \mathrm{~ms}$ until the maximal motor stimulus of plantar flexion and/or foot inversion was found. The same procedure was performed for the common fibular nerve in the immediately medial region of the tendon of the femoral biceps in the popliteal fossa.

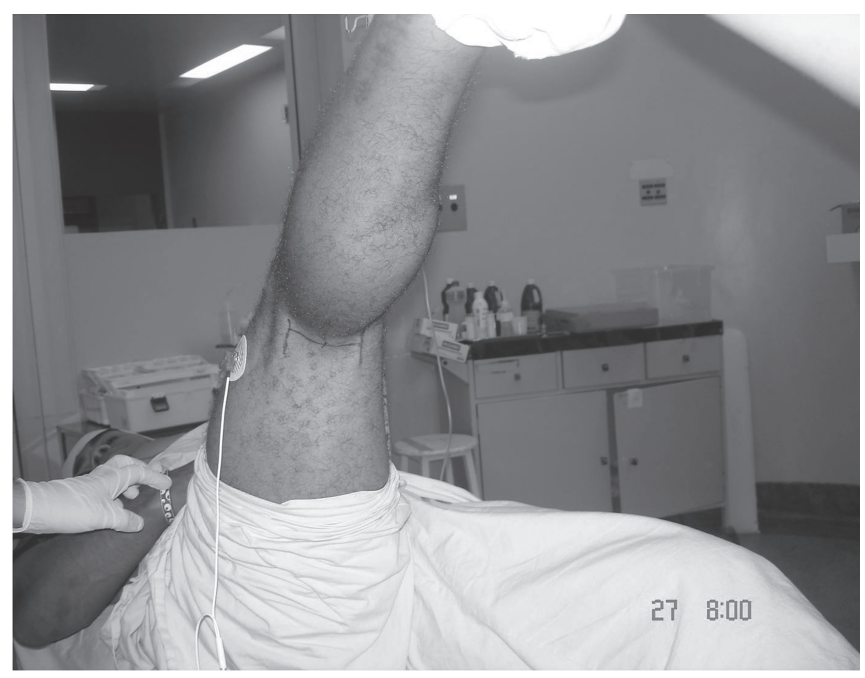

Figure 1 - Positioning for the Blockade and Surface Anatomy of the Popliteal Fossa. Anatomical Reference of the Puncture Site of Tibial and Common Fibular Nerves.
The points of best stimuli were marked and antisepsis was performed. Anesthesia with $1 \%$ lidocaine without vasoconstrictor $(20 \mathrm{mg})$ was applied, and puncture was performed with a short-bevel $5 \mathrm{~cm}$ electrically isolated needle with Teflon (Stimuplex A 50 ${ }^{\circledR}$, B.Braun) connected to the peripheral nerve stimulator (HNS-12 ${ }^{\circledR}$ B.Braun) with a frequency of $2 \mathrm{~Hz}$ and electrical current of $1.0 \mathrm{~mA}$ in the previously marked points. After injection of $10 \mathrm{~mL}$ of $75 \%$ enantiomeric excess $0.35 \%$ levobupivacaine in the tibial nerve point of stimulus, the needle was pulled back and redirected to the point of stimulus of the common fibular nerve until a motor response of dorsal flexion or foot eversion was observed, at which time $10 \mathrm{~mL}$ of $75 \%$ enantiomeric excess $0.35 \%$ levobupivacaine were injected.

All blockades were performed by the authors. The patient was considered ready for surgery when he/she presented complete loss of pinprick sensation on the distribution of the tibial and common fibular nerves.

An independent observer not involved with the blockade noted the times for the location of tibial and common fibular nerves using the percutaneous nerve stimulation from the moment the needle was introduced until the beginning of local anesthetic injection, in addition to the depth of needle insertion in which the electrical stimulation occurred, the latency (from the end of injection until the loss of pinprick sensitivity), and efficacy of the blockade in the region of the tibial, sural, deep fibular, and superficial fibular nerves. Results were described as mean and standard deviation.

\section{RESULTS}

One individual, initially selected, was excluded for refusal to participate in the study and he received another anesthetic procedure. Anthropometric data of the 28 patients are described in Table I.

In the 28 individuals who participated in the study, the common fibular an tibial nerves were identified with the response to a minimal stimulus of $0,5 \mathrm{~mA}$.

Table II shows the times to localize the nerves and the depth of the needle for the tibial nerve, while Table III shows the time to perform the blockades and the latency. The time to

Table I - Anthropometric Data of the Studied Patients

\begin{tabular}{ll}
\hline Age (years) & $41.35 \pm 19.76$ \\
Weight $(\mathrm{kg})$ & $71.14 \pm 14.6$ \\
Height $(\mathrm{cm})$ & $161.79 \pm 5.91$ \\
Gender (M/F) (\%) & $12 / 16(43 / 57)$ \\
Physical status ASA & \\
I & $16(57 \%)$ \\
II & $10(36 \%)$ \\
III & $2(7 \%)$ \\
\hline
\end{tabular}

Results expressed as Mean \pm Standard Deviation. 
Table II - Time to Identify the Tibial and Common Fibular Nerves with Percutaneous Nerve Stimulator and Needle Depth to Localize the Tibial Nerve

\begin{tabular}{ll}
\hline $\begin{array}{l}\text { Time to localize the tibial nerve with the } \\
\text { percutaneous nerve stimulator }(\mathrm{sec})\end{array}$ & $57.14 \pm 33.38$ \\
Limits & $10-120$ \\
$\begin{array}{l}\text { Time to localize the common fibular nerve with } \\
\text { the percutaneous nerve stimulator }(\mathrm{sec})\end{array}$ & $32.85 \pm 30.61$ \\
Limits & $10-130$ \\
Needle depth for the tibial nerve $(\mathrm{cm})$ & $10.71 \pm 3.07$ \\
Limits & $7-19$ \\
\hline
\end{tabular}

Results expressed as Mean \pm Standard Deviation.

Table III - Time to Perform and Latency of Tibial and Common Fibular Nerves Block Using Peripheral Nerve Stimulator

\begin{tabular}{ll}
\hline Time to perform tibial nerve block (min) & $2.22 \pm 1.20$ \\
Limits & $1-3.5$ \\
Time to perform common fibular nerve block (min) & $1.79 \pm 1.14$ \\
Limits & $0.5-4$ \\
Latency of the tibial nerve blockade (min) & $15.46 \pm 5.72$ \\
Limits & $9-25$ \\
Latency of the common fibular nerve blockade & $12.07 \pm 5.13$ \\
(min) & $5-22$ \\
Limits & \\
\hline
\end{tabular}

Results expressed as Mean \pm Standard Deviation.

perform the blockades takes into account the moment the needle was introduced until the injection of the anesthetic, while the latency refers to the time between the end of the injection until loss of pinprick sensitivity.

The time for the percutaneous identification of the tibial nerve was 57.1 seconds, while that of the common fibular nerve was 32.8 seconds. The mean depth of needle insertion into the tibial nerve was $10.7 \mathrm{~mm}$, but in an obese patient it was $19 \mathrm{~mm}$.

Tourniquet was used in ten patients since the surgical procedure required. Tourniquet was not used in patients undergoing amputation and debridement as well as in those undergoing surgeries for removal of synthesis material and hallux valgus.

In patients who underwent ankle arthroscopy the blockade was associated with that of the femoral nerve. In 12 patients there was the need of saphenous nerve block, since the area to be operated involved its sensorial branches.

Surgical procedures performed in this study are in Table IV.

Blood aspiration, paresthesia, clinical signs of anesthetic intoxication, or hemodynamic compromise during or after the technique for the blockade of the sciatic nerve in the popliteal region were not observed. Sensorial or motor deficit after complete recovery from the blockade was not observed.
Table IV - Surgeries Performed

\begin{tabular}{ll}
\hline Types of Surgery & $\mathrm{n}=28$ \\
Toe amputation & 2 \\
Forefoot amputation & 2 \\
Ankle arthroscopy & 2 \\
Biopsy of phalangeal tumor & 1 \\
Debridement of diabetic foot & 4 \\
Correction of talus fracture & 2 \\
Removal of calcaneus synthesis material & 5 \\
Metacarpal fracture & 4 \\
Hallux valgus & 5 \\
Metacarpal osteotomy & 1 \\
\hline
\end{tabular}

\section{DISCUSSION}

Regional peripheral block techniques have been gaining more focus due to the hemodynamic stability, postoperative analgesia, and the possibility to be used in outpatient surgeries ${ }^{1,2}$. The use of peripheral nerve stimulator with electrically isolated needles facilitates the identification of nerve branches and allows the development of techniques aiming at greater patient comfort ${ }^{18}$.

The development of new resources, such as the percutaneous nerve stimulator, allows percutaneous nerve exploration before needle insertion, allowing the identification of peripheral nerve branches anatomical variations ${ }^{11,12}$. Studies have used ultrasound to identify the sciatic nerve in the popliteal fossa ${ }^{13-17}$. In these studies, images were obtained proximal to the popliteal fossa showing the tibial and common fibular nerves as round hyperechoic structures superficial and lateral to the tibial artery, facilitating the introduction of the needle and identification of the nerves by the peripheral nerve stimulator $13,14,17$.

Knowing the anatomy of the popliteal region allows a more safe and simple approach of the tibial and common fibular nerves with a single puncture point due to their superficiality. The literature shows a wide anatomical variation on the level of division of the sciatic nerve, which in some cases is responsible for reports of failure in one of the components (tibial or common fibular nerve) in regional techniques ${ }^{6,7}$. In this study, the use of percutaneous nerve stimulator facilitated the early identification of the desired nerve branches, and a stimulus was observed in all cases with a mean execution time which was lower than one minute demonstrating the effectiveness and ease implementation of this technique.

According to Winnie, regional anesthesia is mainly an exercise in applied anatomy ${ }^{19}$. The sciatic nerve consists of two components, the common fibular and tibial nerves, which are involved by a common connective tissue (sheath) forming a single trunk. The division in two branches can occur at variable levels, as has been shown by several authors $6-8,13,14$. Vloka et al. ${ }^{6}$ analyzed the anatomical implications for blockades in the popliteal fossa according to the division of the 
sciatic nerve and they related the variations to occasional failures. Analyzing 14 corpses they observed that using 5, 7, and $10 \mathrm{~cm}$ long needles proximally to the popliteal fold there is a $46 \%, 57 \%$, and almost $100 \%$ chance, respectively, of being proximal to the division of the sciatic nerve. This demonstrates that traditional techniques 4,5 that recommend the approach 6 to $7 \mathrm{~cm}$ above the fold are associated with a considerable chance of failure ${ }^{6-8}$.

The discussion on the need of finding one or two stimuli with the peripheral nerve stimulator for the blockade is also controversial ${ }^{7,8}$. Xavier et al. ${ }^{20}$ demonstrated that when the puncture is performed as proximally as possible to the popliteal fold $(10 \mathrm{~cm})$, a difference in the latency and in the number of failure was not observed when a single stimulus was compared to double stimuli. In this study, the mean needle insertion was $10.7 \mathrm{~mm}$ after stimulation of the region of the tibial nerve and after the injection of the anesthetic it was redirected superficially to the point for the common fibular nerve and the remaining of the medication was injected.

Failures were not observed with the approach described here because at the level investigated the division of the sciatic nerve had already occurred in all cases ${ }^{6,7}$. The superficiality of the structures, confirmed by the ultrasound, is another advantage allowing prior stimulus with the percutaneous nerve stimulator; therefore, greater precision is obtained when searching for the stimulus with the needle connected to the neurostimulator.

The use of ultrasound has been gaining space in peripheral nerve blocks in the last ten years ${ }^{13}$. The use of this resource has great advantages in relation to the traditional technique, such as direct visualization of nerves and proximal vascular structures, confirmation of local anesthetic deposition around the nerves, and reduction of complications, such as intraneural and intravascular injection of local anesthetics.

Seen on the ultrasound, the popliteal fossa especially at the level of the popliteal fold where the approach described here was performed shows the tibial and common fibular nerves as superficial structures. Popliteal vessels are shown as deep and medial structures in relation to the tibial nerve. The lateral head of the gastrocnemius muscle, which is interposed between the tibial and common fibular nerves, is the reference to be used in the ultrasound approach (Figure 2).

Peripheral nerve blocks with the neurostimulator allow the determination of the exact location of the nerve without the need of looking for paresthesias ${ }^{21}$. The single injection associated to peripheral stimulation is related to a higher success rate of the blockade. There are virtually no failure when the injection is performed after the induction of muscular contractions with an electrical current smaller than $0,5 \mathrm{~mA}$, as it has been demonstrated in a prior study by Fonseca et al. ${ }^{18}$ and confirmed in the present study. We observed that isolated muscular calf contractions can lead to blockade failure and it is not the correct stimulus, since in a study with cadavers we observed muscular branches of the tibial nerve (Figure 3).

Among the patients in this study four had peripheral vasculopathy due to pathological complications especially diabetes, and therefore they were chronically ill patients on different

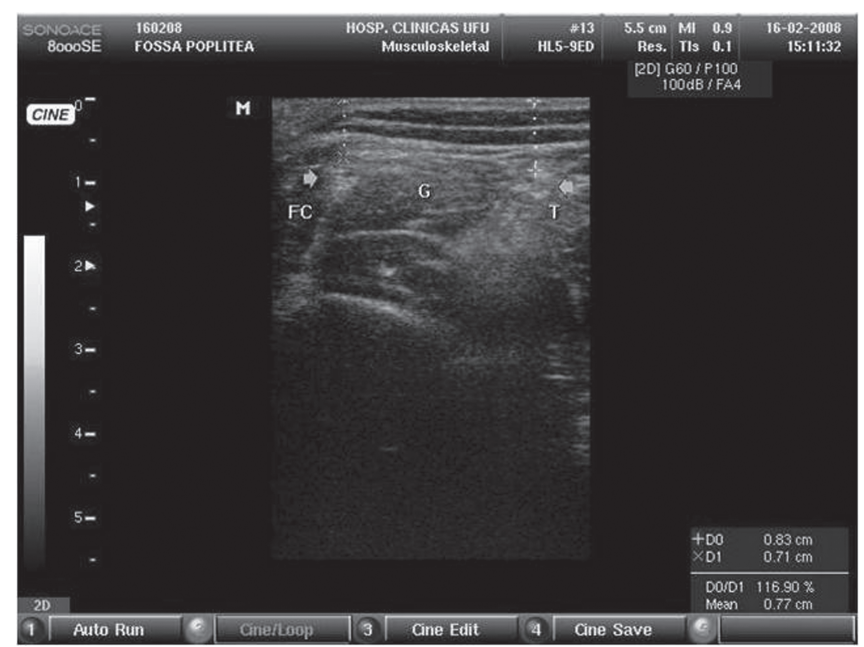

Figure 2 - Ultrasound References to Localize the Tibial (T) and Common Fibular (FC) Nerves. Note the Lateral Head of the Gastrocnemius Muscle (G) Interposed between the Nerve Structures. On the Right Lower Corner, the Depth of the Nerve.

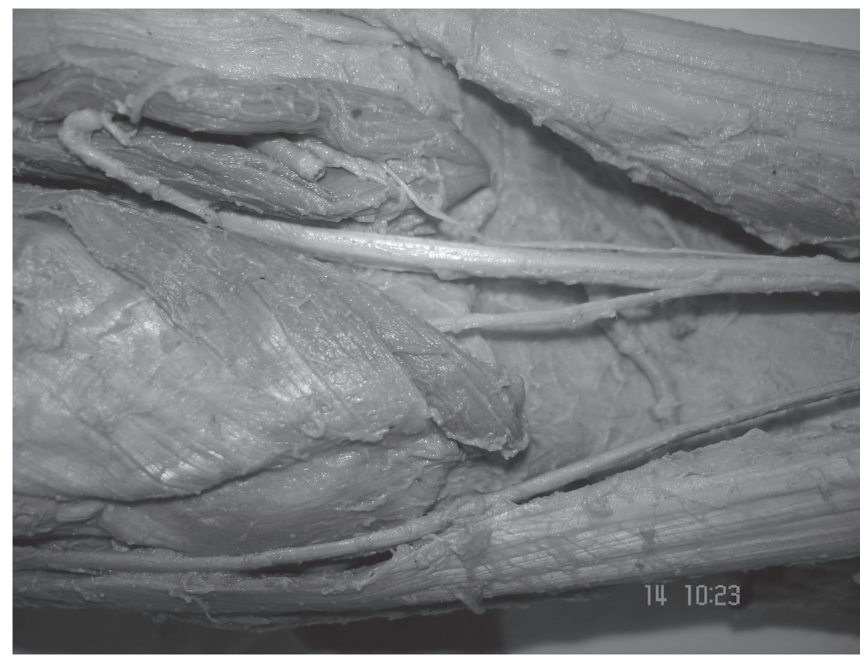

Figure 3 - Cadaver Study at the Human Anatomy Laboratory of the Universidade Federal de Uberlândia. Dissection of the Popliteal Fossa. The Femoral Biceps (Laterally) and Semitendinosus (Medially) Muscles were Separated to Visualize the Tibial and Common Fibular Nerves. Note the Presence of the Muscle Branch Coming out of the Tibial Nerve.

treatments due to multiple organ involvement. In these patients, tibial and common fibular nerve block was useful especially in those with important compromise of the cardiorespiratory system due to the little manipulation and negligible hemodynamic repercussions, since changes in these parameters associated with the anesthetic technique used were not observed 22.

Fanellli et al. ${ }^{21}$ demonstrated low patient acceptability of techniques with multiple injections with the aid of a peripheral nerve stimulator in different techniques of sciatic nerve block in which the identification of both branches of the sciatic nerve 
was investigated. In our study, a single puncture was used to reduce patient discomfort and improve patient satisfaction. This new approach has proven to be easy to perform without failures, in addition to the ease identification of the reference points with the use of percutaneous nerve stimulator.

The large diameter of the sciatic nerve can make the latency of the blockade less predictable than in other peripheral blocks. Although latency is influenced by the chemical properties of the anesthetic used, such as concentration and volume ${ }^{11}$, other factors also interfere with it, especially the type of motor response obtained with the neurostimulator ${ }^{23,24}$ or the intensity of the current ${ }^{23-27}$. The mean latency observed for the tibial and common fibular nerve was 15 and 12 minutes, respectively, which is considered normal due to the diameter of these nerves.

The knowledge of the anatomy in regional anesthesia, along with new technological resources, provides safer and more effective approaches with greater patient satisfaction, represented by the low index of failures and complications. The approach of the tibial and common fibular nerves with single puncture in the popliteal fossa using percutaneous nerve stimulator is a great option for anesthesia and analgesia in foot surgeries.

Further comparative studies of this new approach with traditionally described techniques, as well as ultrasound-guided blocks, can be developed. 